Research Journal of Applied Sciences 6 (7-12): 479-482, 2011

ISSN: $1815-932 \mathrm{X}$

(C) Medwell Journals, 2011

\title{
Effect of BCP Layer and Silver Cathode on the Optimization of the Electric Field in P3HT BHJ Organic Solar Cells
}

\author{
${ }^{1}$ M.R. Merad Boudia, ${ }^{1}$ A.M. Ferouani, ${ }^{2}$ A. Cheknane and ${ }^{1}$ B. Benyoucef \\ ${ }^{1}$ Unit of Research Materials and Renewable Energies, \\ University Abou Baker Belkaid of Tlemcen, Tlemcen, Algeria \\ ${ }^{2}$ Laboratory of Study and Development of Semiconductors and Dielectric Materials, \\ University Amar Telidji of Laghouat, Laghouat, Algeria
}

\begin{abstract}
The efficiency of organic solar cells can be increased by stacking several OPVCs and connecting them in series to optimize the absorption of the incident light. In this study, researchers report the results on the optical modelling of a multi-layer organic photovoltaic device in which the incident light of sun is absorbed in the active layer. The influence of the optical parameters and thicknesses of different layers had been taken into account to improve the device performance. A composites of poly (3-hexylthiophene) (P3HT)/6, 6-Phenyl C61-Butyric acid Methyl ester (PCBM) blends are used as photo-active materials, sandwiched between a transparent Indium Tin Oxide (ITO)-electrode and a metallic $(\mathrm{Ca}, \mathrm{Al}, \mathrm{Ag})$ backside contacts. This study aims to show optical effects of an extra interfacial layer of poly (3, 4-ethylenedioxythiophene)/(poly (styrenesulfonate) (PEDOT/PSS) on top of the ITO-electrode and a BCP layer on the metallic electrode. The objective is the use of some recent methods which make it possible the description of the electric field distribution for the organic solar cells described previously with different deposited layers to make a rigorous modelling of these devices. The basic criterion for optical optimization is to maximize the energy absorption in the active layer according to the distribution of electrical field on the device to enhancing the dissociation rate.
\end{abstract}

$\underline{\text { Key words: Organic solar cells, optical modelling, refractive index, P3HT/PCBM, energy absorption, device }}$

\section{INTRODUCTION}

In these last years several research was undertaken on the study of the interaction between the incident light with the photovoltaic devices of organic semiconductors. The modelling of optical absorption in organic solar cells has a great importance in the prediction of the electro-optical properties of these devices because the dissociation of charges is assisted strongly by the application of an electric field in organic materials.

Monestier et al. (2007) have presented a method of optimization of the electromagnetic field in the organic solar cells and an electro-optical modelling of these cells describing the generation rate of excitons based on the calculation of the profile of absorbed energy. Filppov and Serebryakova (2007) presented in a concrete analysis of interference effects on the spatial field distibution. Another research group (Moule and Meerholz, 2007a, b) made the minimization of the optical losses in the bulk heterojunction solar cells by using the complex index of refraction and ellipsometry. The electromagnetic properties of reflection, transmission and absorption have an importance in the optical characterization of multi-layer system used in inorganic and organic photovoltaic devices. They describe the interaction of the incident light with the various layers of the system. Depending on optical constants and thicknesses of mono-layers we use here a matrix transfer method (Petterson et al., 1999; Hecht, 2002) to calculate the electromagnetic field in the structures of device.

\section{MATERIALS AND METHODS}

In this study researchers present a part of fundamental mechanisms of matter-radiation interaction being given a solid body crossed by a sinusoidal monodirectionnal wave being propagated on $\mathrm{OZ}$ axis, we have two cases.

Case of a double-layered system: When the light travels of the first medium with $n_{1}$ index of refraction in the second medium with $\mathrm{n}_{2}$ index of refraction, the amplitudes of the electric field of the electromagnetic wave reflected and transmitted of the interface are governed by the coefficients of Fresnel (Li, 2003) (Fig. 1).

Corresponding Author: M.R. Merad Boudia, Unit of Research Materials and Renewable Energies, 


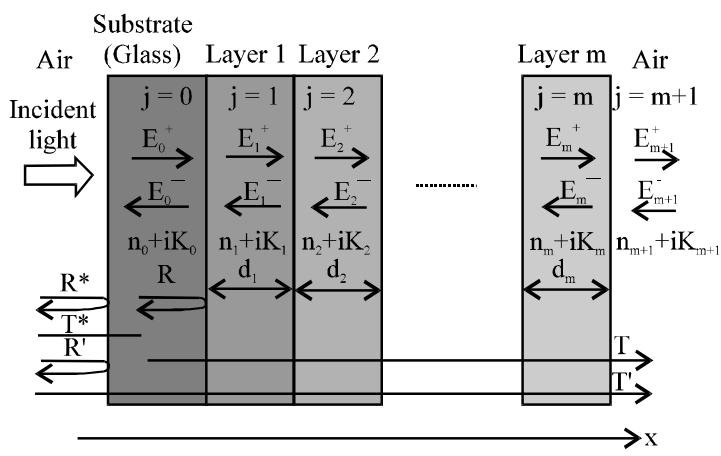

Fig. 1: Propagation of the light in a double-layered system

The Fresnels coefficients are defined as being the relationship between the amplitudes of the electric field before and after an interface. In the case of a system of two mediums $i$ and $i+1$, the reflection of a beam with normal incidence on the interface gives the coefficient of Fresnel:

$$
\mathrm{r}=\mathrm{E}_{\mathrm{e}} / \mathrm{E}_{\mathrm{i}}
$$

where, $E_{e}$ and $E_{i}$ are the electric fields after and before an interface, respectively. For linear, isotropic and homogeneous mediums, researchers can calculate the coefficients of Fresnel directly with the indices of refraction. If the angle of incidence of the beam on the medium is null, we has:

$$
\begin{aligned}
& \mathrm{r}_{\mathrm{TE}}=\frac{\mathrm{n}_{1}-\mathrm{n}_{2}}{\mathrm{n}_{1}+\mathrm{n}_{2}}, \mathrm{t}_{\mathrm{TE}}=\frac{2 \mathrm{n}_{1}}{\mathrm{n}_{1}+\mathrm{n}_{2}} \\
& \mathrm{r}_{\mathrm{TM}}=\frac{\mathrm{n}_{2}-\mathrm{n}_{1}}{\mathrm{n}_{1}+\mathrm{n}_{2}}, \quad \mathrm{t}_{\mathrm{TM}}=\frac{2 \mathrm{n}_{1}}{\mathrm{n}_{1}+\mathrm{n}_{2}}
\end{aligned}
$$

Case of a multi-layer system: Two methods of calculation are possible:

- A method where we wrote incident, reflected and transmitted fields, layer by layer and we specify that the boundary conditions of these fields must be satisfied (Gruber et al., 2005)

- The TMM introduced by Petterson et al. (1999) that researchers use after here: the multiplication of the matrices will describe how the wave propagates by the whole device. Figure 1 shows the arrangement of propagation of the forwards and towards fields in the back of the device under AM 1.5 solar spectrum demonstrated

The Eq. 4 gives the sum of the electric field in the positive and the negative directions respectively:

$$
E_{j}(x)=E_{j}^{+}(x)+E_{j}^{-}(x)
$$

Since, optical energy is a measurable quantity, the first stage in the calculation of the optical generation rate is to calculate the Poynting vector brought back to an average per hour:

$$
\left\langle\mathrm{P}_{\mathrm{j}}\right\rangle=\frac{1}{2}\left[\mathrm{E}_{\mathrm{j}} \times \mathrm{H}_{\mathrm{j}}^{*}\right]
$$

The time average of the energy dissipated per second in layer $\mathrm{j}$ at position $\mathrm{x}$ is given by:

$$
\mathrm{Q}_{\mathrm{j}}(\mathrm{x})=\frac{1}{2} \mathrm{c} \varepsilon_{0} \alpha_{\mathrm{j}} \eta_{\mathrm{j}}\left|\mathrm{E}_{\mathrm{j}}(\mathrm{x})\right|^{2}
$$

Where:

$\mathrm{c}=$ The speed of light

$\varepsilon_{0}=$ The permittivity of free space

$\eta_{j}=$ The refractive index

The absorption coefficient is:

$$
\alpha_{j}=\frac{4 \pi \mathrm{k}_{\mathrm{j}}}{\lambda}
$$

The relations managing electric and magnetic fields and which allow the direct passage of a layer to other are given by Hecht (2002):

$$
\left|\begin{array}{c}
E_{j-1} \\
H_{j-1}
\end{array}\right|=\left|\Psi_{m}\right| \begin{aligned}
& E_{j} \\
& H_{j}
\end{aligned} \mid
$$

Where, $\Psi_{\mathrm{m}}$ is a $2 \times 2$ matrix which contains the permittivity values for each medium. The rate equation for the charge pair population is given as:

$$
\frac{\mathrm{dX}}{\mathrm{dt}}=\mathrm{G}-\mathrm{X}\left(\mathrm{K}_{\mathrm{f}}+\mathrm{K}_{\mathrm{d}}\right)+\mathrm{R}
$$

Where:

$\mathrm{K}_{\mathrm{d}}$ and $\mathrm{K}_{\mathrm{f}}=$ The dissociation and decay rates, respectively

$\mathrm{R}=\gamma \mathrm{np}$ is the bimolecular recombination rate

$\gamma=$ The Langevin recombination parameter

The dissociation rate in the presence of an electric field given is by Onsager theory as:

$$
\mathrm{K}_{\mathrm{d}}(\mathrm{E})=\frac{3<\mathrm{u}>\mathrm{e}}{4 \pi \varepsilon \varepsilon_{0} \mathrm{a}^{3}} \exp \left(\frac{-\Delta \mathrm{E}}{\mathrm{KT}}\right)\left\{1+\mathrm{b}+\frac{1}{3} \mathrm{~b}^{2}+\frac{1}{18} \mathrm{~b}^{3}+\ldots\right\}
$$


When:

$$
\mathrm{b}=\frac{\mathrm{e}^{3} \mathrm{E}}{8 \pi \varepsilon \varepsilon_{0} \mathrm{~K}^{2} \mathrm{~T}^{2}}
$$

is the field parameter. The charge efficiency for individual charge pairs as introduced by Braun (1984):

$$
P=\frac{K_{d}}{K_{d}+K_{f}}
$$

\section{RESULTS AND DISCUSSION}

Researchers can apply the model described previously to organic solar cells Fig. 2 composited of

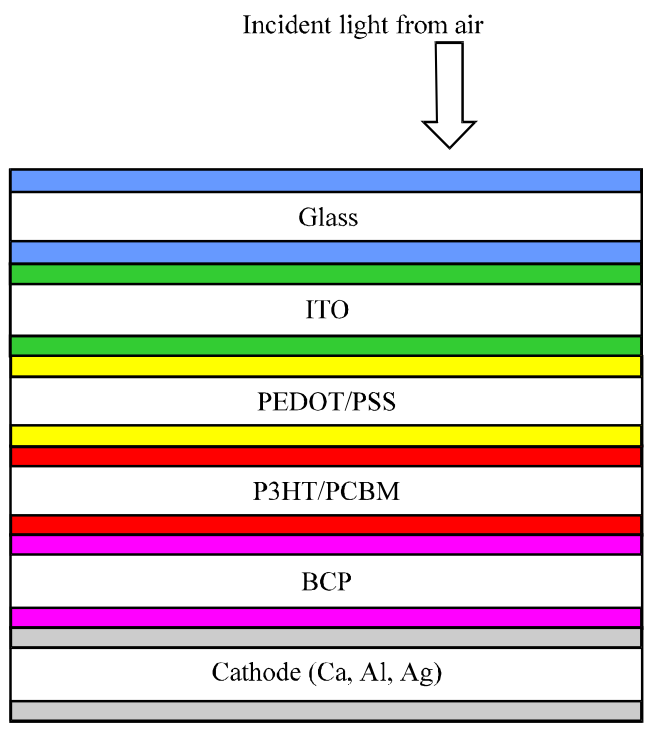

Fig. 2: Diagram of the studied an organic solar cell

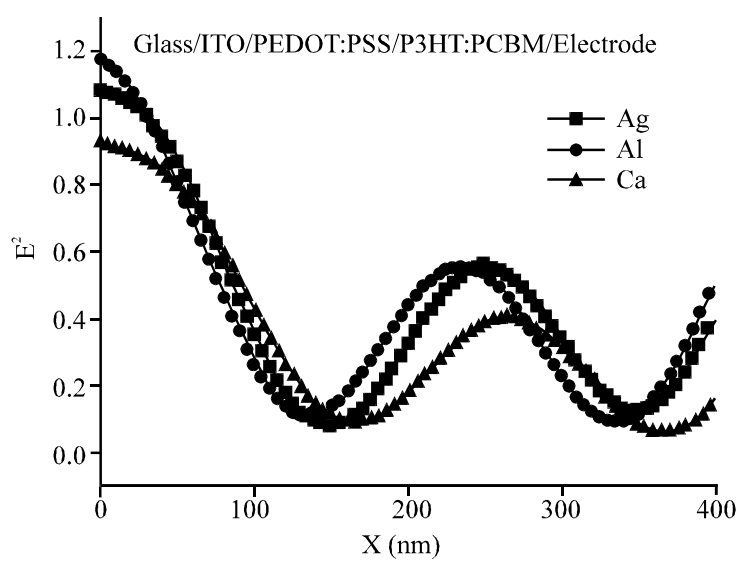

Fig. 3: Square of the electrical field in the active layer in $\mathrm{P} 3 \mathrm{HT} / \mathrm{PCBM}$ of an organic solar cell with different electrode layers in $\mathrm{Ag}, \mathrm{Al}$ and $\mathrm{Ca}$
Glass/TTO/(PEDOT-PSS)/(P3HT/PCBM)/(Ca, Al, Ag) as shown in Fig. 3. The thickness of ITO anode is supposed $\mathrm{d}=140 \mathrm{~nm}$, layer PEDOT/PSS with also a thickness of $140 \mathrm{~nm}$, the back contact has generally a thickness of $30 \mathrm{~nm}$, this last material is assumed to be variable and reflective in the simulations which is the most dominant boundary condition with the various thicknesses of the active layer. Bathocuproine or 2, 9Dimethyl-4, 7-diphenyl-1, 10-Phenanthroline (BCP) is used as a passivation layer to prevent excitons generated in the active layer from being quenched at the organic/metal interface and to protect the donor/acceptor layer during metal deposition. Optical constants of ITO, PEDOT-PSS, BCP films were obtained from ref. (Filppov and Serebryakova, 2007), the optical constants of P3HT/PCBM were obtained from ref. (Monestier et al., 2007) and $\mathrm{Ca}, \mathrm{Al}$ and $\mathrm{Ag}$ were obtaind from ref. (Pandey et al., 2009).

Figure 3 and 4 show the profile of the electric field in each layer of the photovoltaic device according to the thickness of the active layer. Firstly, researchers has choosing BHJ solar cells because there represents the most recent efficient devices. Then, we have proved by numerical simulations that the use of Silver materials increases the electric field on the active layer near the metallic electrode.

The protective coating in PEDOT/PSS affects clearly the luminous energy transmitted in the active layer when this energy is decreased for very thinner active layers because there is a big region of reflections at the counter electrode in $\mathrm{Al}$ and $\mathrm{Ag}$ since, the use of $\mathrm{BCP}$ layer as a hole transport layer enhance and increase the electric field

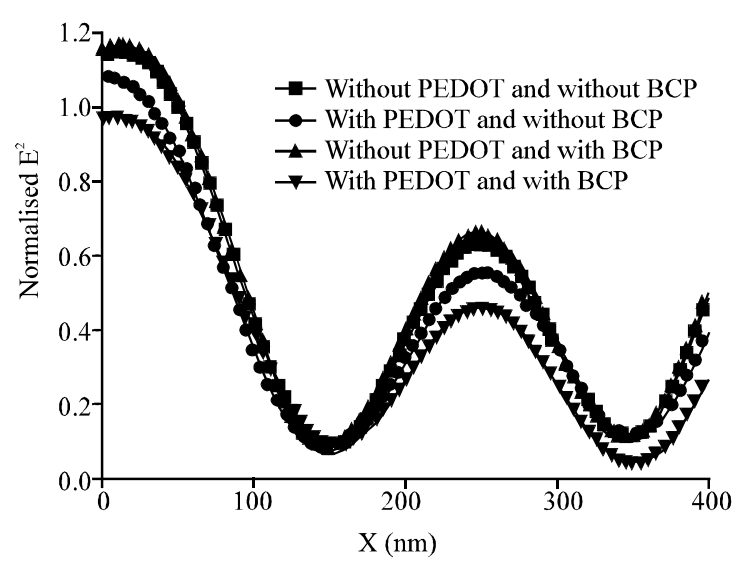

Fig. 4: Square of the electrical field in the active layer in P3HT/PCBM of an organic solar cell with and without protective and buffer layers and metallic electrode in silver 


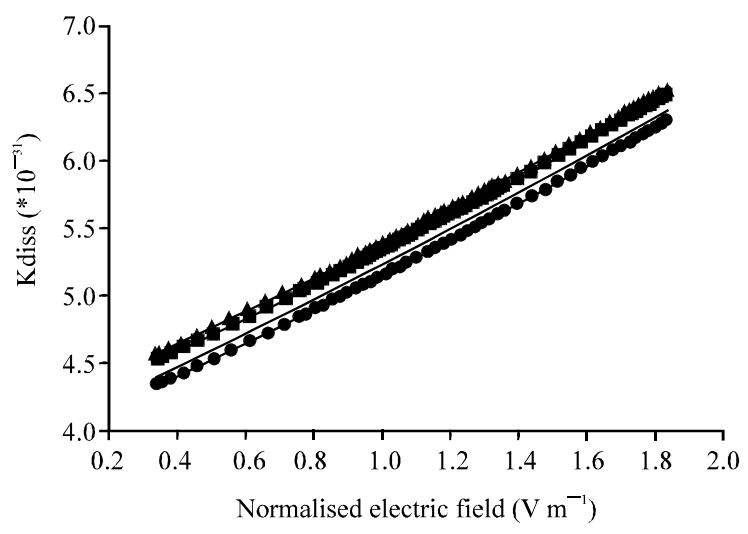

Fig. 5: Dissociation rate in the presence of an electric field given by Onsager theory

distribution on the active region. The dissociation rate of a geminate charge pair in found to be a key parameter in determining the device performance. Figure 5 shows the proportionality between this last parameter and the electric field distribution inside the devices.

\section{CONCLUSION}

In this study, the performances of organic solar cells devices with and without PEDOT-PSS, BCP as protective, blocking layers respectively and (aluminium, silver and calcium) as back contacts were investigated and compared by an optical modelling.

Reseachers presented in this study the results of modelling and digital simulation which describe the evolution of the magnetic and electric fields in bulk heterojunction solar cells as well as the optimization of the electric field distribution according to the thickness of the active layer.

From the results reported here for the 1st hand it is clear that the solar cells in P3HT/PCBM as active layers was a very hight distribution of electric field with silver electrodes, aluminum is a second optimization material and calcium is a poor material to be used as metallic electrode.

For the 2nd hand it is clear also that the solar cells without protective layer in PEDOT/PSS and with BCP layer was an optimum distribution of electric field, solar cells without protective and BCP layers was taken the second place, solar cells with protective and without $\mathrm{BCP}$ layers was taken the third place and finally the solar cells with protective and $\mathrm{BCP}$ layers was taken the last place. The results of simulation are in good agreement with those published in the literature by the use of this recent method. In this study researchers applied this sofisticated method to enhance the performance of photovoltaic devices.

\section{REFERENCES}

Braun, C.L., 1984. Electric-field assisted disociation of charge stades as a mechanism $\mathrm{f}$ photocarrier production. J. Chem. Phys., 80: 4157-4161.

Filppov, V.V. and L.M. Serebryakova, 2007. Optical characteristics of a multilayer photovoltaic cell for oblique incidence of light. J. Appl. Spectrosc., 74: 884-891.

Gruber, D.P., G. Meinhardt and W. Papousek, 2005. Modelling the light absorption in organic photovoltaic devices. Solar Energy Mater. Solar Cells, 87: 215-223.

Hecht, E., 2002. Optics. 4th Edn., Addison-Wesley, USA., ISBN-13: 9780321188786 , Pages: 698.

Li, L., 2003. The design of optical thin film coatings with total and frustrated total internal reflection. Opt. Photonics News, 14: 24-30.

Monestier, F., J.J. Simon, P. Torchio, L. Escoubas and F. Flory et al., 2007. Modeling the short-circuit current density of polymer solar cells based one P3HT: PCBM blend. Solar Energy Mater. Solar Cells, 91: 405-410.

Moule, A.J. and K. Meerholz, 2007a. Interference method for the refractive determination of the complex index of thin polymer layers. Appl. Phys. Lett., Vol. 91.

Moule, A.J. and K. Meerholz, 2007b. Minimizing optical losses in bulk heterojunction polymer solar cells. Appl. Phys., 86: 721-727.

Pandey, A.K., P.E. Shaw, I.D.W. Samuel and J.M. Nanzi, 2009. Effect of metal cathode reflectance on the exciton-dissociation effiency in heterojunction organic solar cells. Appl. Phys. Lett., Vol. 94.

Petterson, L.A.A., L.S. Roman and O. Inganas, 1999. Modeling photocurrent action spectra of photovoltaic devices based on organic thin films. J. Appl. Phys., 86: 487-496. 